\title{
Das Ellwanger Katalogisierungsprojekt
}

Ende September 1992 konnte in der Württembergischen Landesbibliothek Stuttgart die von der DFG im Rahmen des Programms „Erschließung von Spezialbeständen“ finanzierte Katalogisierung der Altbestände des Ellwanger Peutinger-Gymnasiums abgeschlossen werden. Seit dem 01.10.1990 hatte eine Diplom-Bibliothekarin (Vergütungsgruppe BAT $\mathrm{Vb}$ ), die über hervorragende Kenntnisse in den alten Sprachen verfügt, die partienweise nach Stuttgart transportierten Bände per EDV im Südwestdeutschen Bibliotheksverbund (SWB) katalogisiert. Ebenso wie die bereits 1991 beendete Erschließung des Rottweiler Albert-Magnus-Gymnasiums ${ }^{1}$ wurde das Projekt vom Zentralkatalog Baden-Württemberg, einer Hauptabteilung der Württembergischen Landesbibliothek, betreut.

Die Bedeutung der Bibliothek des Peutinger-Gymnasiums ist eng mit der Geschichte der Stadt Ellwangen und des von Jesuiten 1658 begründeten Gymnasiums verknüpft. ${ }^{2}$ Als Kern der Bibliothek sind die Bestände der Jesuitenschule anzusehen. Noch im 17. Jahrhundert kamen einige kleinere Privatbibliotheken und Bücherspenden hinzu, ehe der Stiftsdekan Desiderius von Peutingen 1718 seine private Büchersammlung der Jesuitenschule, die heute seinen Namen trägt, überließ. Aus den Beständen der berühmten „Bibliotheca Peutingeriana“ seines Vorfahren Konrad Peutinger, die nach Augsburg gegangen war, kamen 1725 zahlreiche dort nicht benötigte Bücher nach Ellwangen zurück. Auch Bücher aus den Jesuitenkollegien in Dillingen und Ingolstadt sind in der Bibliothek des PeutingerGymnasiums nachweisbar. Nach dem Anschluss Ellwangens an Württemberg wurden die Bestände der alten Ellwanger Bibliotheken (Stiftskapitel, Fürstpropstei, Seminar) in einer "Zentralbibliothek" zusammengetragen, die noch weitere säkularisierte Bücherbestände aus Württemberg aufnehmen sollte. Doch es kam anders: 1806 wurde angeordnet, die gesamte Ellwanger Bibliothek mit der Königlichen Bibliothek in Stuttgart zu vereinigen. Mangel an Transportmöglichkeiten, aber vielleicht auch, wie Irtenkauf ${ }^{3}$ andeutet, „passive Resistenz" oder "Schlamperei“ vereitelten diesen Plan. 1808 gelangten nur die Handschriften und einige ältere Drucke nach Stuttgart - „kleine Früchte einer immer wieder einsetzenden Mahnung seitens der Landeshauptstadt". Die verbliebenen circa 109.000 Bände bildeten 1812 wiederum den Grundstock einer neu gegründeten Ellwanger Seminar- und Universitätsbibliothek, die zusätzlich durch Säkularisationsbestände aus Ehingen, Komburg, Rottenburg, Weil der Stadt, Wiblingen und Wiesensteig bereichert wurde. 1818 musste auch diese Bibliothek Bestandsverluste hinnehmen, als nach der Verlegung des Priesterseminars nach Rottenburg und der Angliederung der katholischen Fakultät an die Universität Tübingen circa 3.000 Bände nach Rottenburg und Tübingen verlagert wurden. 1824 übernahm schließlich das Gymnasium die in Ellwangen verbliebenen Bestände. Ein letzter Verlust war 1939 hinzunehmen, als die Württembergische Landesbibliothek weitere 2.769 Bände, darunter Pergamenthandschriften, erhielt (Sign. „Bibl. Elv.“).

Vor dem Hintergrund der hier kurz skizzierten Bestandsgeschichte der heutigen Bibliothek des Peutinger-Gymnasiums wird deutlich, dass sich sowohl Bücher aus

\footnotetext{
1 Horst Hilger und Daniela Maisch. „Altbestände in Gymnasialbibliotheken: Das Beispiel des Rottweiler Katalogisierungsprojekts“. BIBLIOTHEKSDIENST 24 (1990) H.1. S.23-33

2 Eine ausführliche Darstellung der Geschichte bringt Heribert Hummel. „Habent sua fata libelli: Zur Geschichte der Ellwanger Gymnasialbibliothek“. In: Festschrift zum 325jährigen Jubiläum. Ellwangen 1983. S.65-80

${ }^{3}$ Wolfgang Irtenkauf. „Alte Bibliotheken in Ellwangen“. In: Ellwanger Jahrbuch 20 (1962-64) S.54-77
} 
den alten Ellwanger Bibliotheken, insbesondere der fürstpröpstlichen und der Jesuitenbibliothek, als auch - allerdings in geringerem Umfang Säkularisationsbestände aus Wiblingen, Ehingen, Komburg etc in der Sammlung befinden. Die verschiedenen Provenienzen sind zum größten Teil recht einfach zu erkennen: Bei den Büchern der ehemaligen Jesuitenbibliothek ist das untere Feld des Buchrückens schwarz markiert. Bücher der fürstpröpstlichen Hofbibliothek tragen gewöhnlich am oberen Rand des Titelblatts den Vermerk „Ex Bibliotheca aulica Reverendissimi et Illustrissimi Principis Elvacensis“. Zahlreiche Bände tragen die Wappenstempel von Ellwanger Fürstpröpsten auf dem Vorderdeckel. Einige Exlibris verweisen auf die Privatbibliothek des regierenden Fürstpropsten, so schöne Kupferstich-Exlibris des Propsten Anton Ignaz von Fugger mit zusätzlichen handschriftlichen Vermerken. Bücher aus der Bibliothek des Franziskanerklosters zu Ehingen haben teilweise rot gefärbte Buchrücken, und Bände des Ritterkantons Odenwald sind leicht an einem blauen Exlibris mit dem Wappen des Ritterkantons auf dem Innendeckel zu identifizieren.

Der Gesamtbestand der Bibliothek beläuft sich auf circa 15.000 Bände. Der historische Teil, der katalogisiert worden ist, umfasst auf der Grundlage einer Hochrechnung für das Handbuch der historischen Buchbestände circa 1.500 Titel in etwa 4.300 Bänden. Fast zwei Drittel der circa 1.500 Titel sind aus dem 16. bis 18. Jahrhundert (oder ohne Erscheinungsjahr). Im gesamten Projektzeitraum wurden über die Online-Verbindung nach Konstanz 4.337 Lokalsätze in den Datenpool des SWB eingegeben, davon 2.735 (= $63 \%$ ) Neuaufnahmen, also Lokalsätze von noch nicht im SWB nachgewiesenen Titeln. In der Schule ist zusätzlich eine umfangreiche und vollständig unerschlossene Sammlung von mehreren tausend, nach Orten/Schulen gebündelten Programm-Abhandlungen (Schulschriften) deutscher Lehranstalten des Zeitraums 1840 bis Anfang des 20. Jahrhunderts vorhanden. Schon auf den ersten Blick erstaunt der hohe Anteil von Schulschriften mittel- und ostdeutscher Provenienz. Diese Sammlung konnte für das Erschließungsprojekt nicht berücksichtigt werden.

Vor Projektbeginn war nur circa ein Drittel der Altbestände durch einen unzureichenden, vorwiegend handschriftlich vorliegenden alphabetischen Zettelkatalog erschlossen. Für den größten Teil des Altbestandes existierte kein Katalog. Da der Altbestand obendrein vollkommen ungeordnet aufgestellt war, konnte man auf einzelne Titel nur mit großem Suchaufwand zugreifen. Im Rahmen der Katalogisierungsarbeiten wurde jetzt auch dieser Teil des Altbestandes komplett geordnet und mit Signaturen versehen. Nach Abschluss des Projekts stehen folgende Nachweisinstrumente zur Verfügung:

- als lokale Nachweisinstrumente in der Schule ein Alphabetischer und ein Systematischer (Zettel-)Katalog, die in der Konstanzer Verbundzentrale ausgedruckt worden sind,

- als regionales Nachweisinstrument die Online-Katalogisate im Datenpool des SWB. Bei den in der Württembergischen Landesbibliothek nach Autopsie katalogisierten Titeln wurde in jedem Lokalsatz die Provenienz - soweit ermittelbar - vermerkt sowie durch einen speziellen Buchstabenschlüssel (ALPR) abrufbar gemacht, 
- (als überregionales Nachweisinstrument die in den nationalen DBIVerbundkatalog übernommenen Titel, die in dessen aktualisierter Form auch bundesweit für Recherchen zur Verfügung stehen). [Anm.: Der letzte Satz ist schon seit Langem überholt]

Die Benutzung der Bibliothek ist in den Räumen der Schule nach Voranmeldung möglich. Arbeitsplätze und Kopiermöglichkeiten sind vorhanden. Alle nach Stuttgart transportierten Bände sind buchpflegerisch behandelt worden. Für Restaurierungsmaßnahmen wurden von der Stadt Ellwangen auf Antrag der Württembergischen Landesbibliothek 6.000,- DM für 1992 bewilligt.

Für die Bibliothek des Peutinger-Gymnasiums in Ellwangen liegt nach Abschluss des DFG-Projekts zum ersten Mal in ihrer Geschichte ein bibliothekarischen Ansprüchen genügendes Katalogsystem vor. Ebenso wie die Sammlungen der Gymnasialbibliotheken in Rottweil, Konstanz, Rastatt, Heidelberg und Offenburg ${ }^{4}$ sind ihre Altbestände durch den regionalen (im SWB) und überregionalen Nachweis (im DBI-VK) auch für die historisch orientierten Wissenschaften zugänglich.

\author{
Horst Hilger \\ (Württembergische Landesbibliothek)
}

aus: BIBLIOTHEKSDIENST 27 (1993) H.1. S.46-49

\footnotetext{
4 Einen Gesamtüberblick über die laufenden Katalogisierungsprojekte in baden-württembergischen Gymnasialbibliotheken gibt Wilfried Sühl-Strohmenger: „Altbestände baden-württembergischer Gymnasialbibliotheken. Beispiele ihrer Erschließung“. In: 81. Deutscher Bibliothekartag in Kassel 1991. Wissenschaftliche Bibliotheken im vereinten Deutschland. Hrsg. von Engelbert Plassmann ... Frankfurt am Main 1992. S.133-149 (Zeitschrift für Bibliothekswesen und Bibliographie; Sonderheft 54)
} 